\title{
Analysis of Wear Track on DLC Coatings after Sliding with MoDTC-Containing Lubricants
}

\author{
Kenji Ohara ${ }^{1)^{*}}$, Kiyoshi Hanyuda'), Yasushi Kawamura ${ }^{1)}, K^{1}$ entaro Omura2), \\ Imari Kameda2), Noritsugu Umehara2) and Hiroyuki Kousaka²) \\ 1)Central Research \& Development Laboratory, Showa Shell Sekiyu K. K., 4052-2 Nakatsu, Aikawa-cho, Aikoh-gun, \\ Kanagawa 243-0303, Japan \\ 2)Department of Mechanical Science and Engineering, Nagoya Univeristy, Furo-cho, Chikusa-ku, Nagoya 464-8603, Japan
}

*Corresponding author: Kenji Ohara (Kenji.Oohara@showa-shell.co.jp)

\begin{abstract}
Wear of DLC coating has been great concern in DLC/Steel sliding surface lubricated by molybdenum dithiocarbamate (MoDTC) containing lubricants. However, in order to design lubrication system with DLC and MoDTC, the mechanism of this issue is needed to be clarified. In this study, the roller-on-disc oscillation friction test by SRV friction tester was conducted to provide the hypothesis about the mechanism of wear of DLC with MoDTC. In friction test, DLC coatings (a-C:H and ta-C) were coated on discs. Counterpart was the SUJ2 bearing steel roller. It was found that MoDTC in lubricants accelerated the wear of the both two DLC coatings and it was dramatic especially for a-C:H coating. In order to clarify the enhancement mechanism of MoDTC for wear of DLC coatings, the surface analysis on DLC coatings and steel roller was conducted with XPS (X-ray Photoelectron Spectroscopy) and surface profile meter. As a result, sp ${ }^{2}$ ratio increased layer was observed on the a-C:H coating with XPS and the surface roughness of wear track was larger than out of wear track after sliding with MoDTC containing lubricant. On the other hand, ta-C did not show the increasing of sp ${ }^{2}$ ratio and the surface roughness of wear track decreased after sliding with and without MoDTC containing lubricant. For both DLC coatings, tribofilm generated from MoDTC was also observed on the counter SUJ2 roller surface with XPS. Depth profile of chemical elements of the tribofilm on the steel roller with XPS contributed to clarify the distribution of Molybdenum compound as $\mathrm{MoO}_{3}$ and $\mathrm{Mo}_{2} \mathrm{C}$. Top surface was $\mathrm{MoO}_{3}$ and the interface between the SUJ2 roller and tribfilm was $\mathrm{Mo}_{2} \mathrm{C}$. The authors provide the hypothetical model about a tribo-chemical reaction between both DLC coatings as ta-C or a-C:H and tribofilm from MoDTC.
\end{abstract}

\section{Keywords}

DLC, wear, lubrication, XPS

\section{Introduction}

Diamond-like carbon (DLC) coatings are well known for their excellent low-friction and wear-resistance [1,2]. Today, they are widely used in various mechanical systems. Especially, fuel efficiency improvement of internal combustion engines is a benfit by using diamond-like carbon (DLC) coating as it owns high hardness and applies low friction of the sliding surface.

Molybdenum dithiocarbamate (MoDTC) as friction modifier additive is widely used in automobile engine lubricants because it has significant advantages in terms of friction reduction with steel/steel sliding surface. However, it is generally accepted that wear of DLC increases by using MoDTC based lubricants. There are several types of DLC in the industry such as tetrahedral amorphous carbon (ta-C) and hydrogenated amorphous carbon (a-C:H). It is said that the brittleness of DLC is increased by the reaction of Molybdenum oxides and a-C:H which is suffering hugely wear under lubrication with MoDTC [3]. On the other hand, several additives in lubricants contributed to decrease wear of DLC under lubrication with MoDTC [4]. However, the mechanism of the DLC wear with MoDTC has been unclear from the viewpoint of what kind of tribo-mechanical phenomena it is based on. Therefore, a guideline to design the lubrication system with DLC and Molybdenum containing lubricants could clearly lead to expand the opportunities of the DLC and lubricants usage. In order to build such guideline, some explanations about tribo-mechanics and tribo-chemistry on surfaces are needed. Hence, the authors investigated the DLC surface after friction test 
and measured the corresponding surface layer which are a consequence of the reaction with MoDTC. This gave insight in the wear phenomenon of the mechanism of DLC wear possible induced by MoDTC.

This paper reports the results of surface analysis by using XPS (X-ray Photoelectron Spectroscopy) technique after SRV sliding under lubrication with a base oil and base oil including MoDTC. The authors conducted roller-on-disc oscillation friction test by SRV friction tester. The material of the roller was SUJ2 (SAE52100). Two types of DLC (ta-C and a-C:H) were deposited on the SUJ2 discs. Surface profile was measured by profilometer. Cross-sectional area of the wear track was calculated from the difference between surface profile out of wear track and surface profile inside of wear track. It was found that MoDTC in lubricants are accelerating the wear of DLC. The authors compared specific wear rate by XPS analysis with the impact of DLC bonding state and chemical elements on surfaces. The authors also proposed a model of wear appearing on DLC in the presence of molybdenum based lubricants.

\section{Experimental method and procedure}

\subsection{DLC coatings and counter material}

Two different types of DLC were prepared, ta-C has 50 GPa hardness and $700 \mathrm{~nm}$ coating thickness. Another type of DLC was a-C:H and got $20 \mathrm{GPa}$ hardness and a coating thickness of $4 \mu \mathrm{m}$. Counter material was steel bearing SUJ2 roller.

\subsection{Lubricants}

API Group III base oil having viscosities of $17.94 \mathrm{~mm}^{2} / \mathrm{s}$ at $40^{\circ} \mathrm{C}$ and $4.06 \mathrm{~mm}^{2} / \mathrm{s}$ at $100^{\circ} \mathrm{C}$ was used as base oil for all tested oils and named as Base in this paper. The MoDTC containing lubricant named Base + MoDTC was formulated as a blend of Base and MoDTC and the concentration was $700 \mathrm{ppm}$ as detection amount of Molybdenum with inductively coupled plasma atomic emission spectroscopy.

\subsection{Friction tester}

The experimental setup is shown in Fig. 1. The roller-on-disc oscillation friction test by SRV friction tester was carried out by machine manufactured by Optimol. Tests were performed on DLC coated SUJ2 disc against steel roller of SUJ2. The roller, measuring $15 \mathrm{~mm}$ in diameter and $22 \mathrm{~mm}$ in length, was loaded

Table 1 The details of DLC films

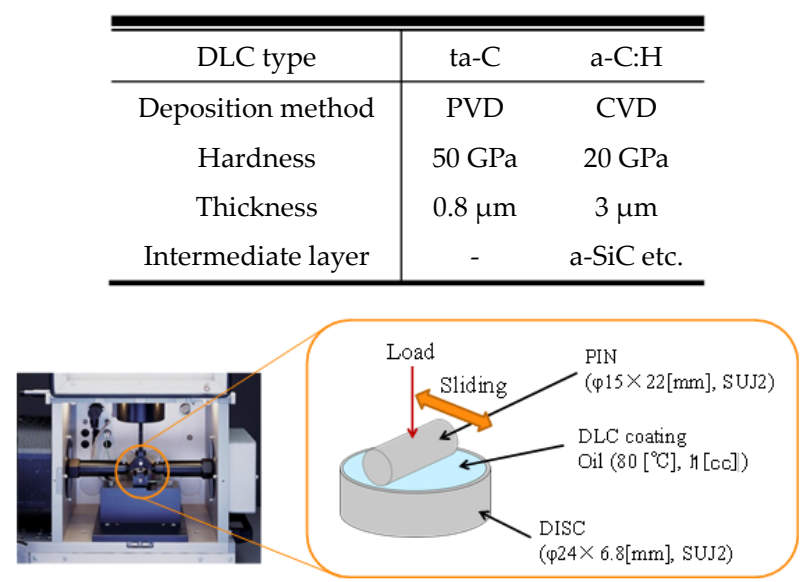

Fig. 1 Schematic image of cylinder-on-disc type tribotester, and outside picture of cylinder and disc and rubbed against DLC coated disc. DLC films were coated on disc so that cross sectional surface and wear depth can be measured more accurately than if roller was coated. Both roller and disc were lubricated with $1 \mathrm{cc}$ lubricants. Disc was heated to $80^{\circ} \mathrm{C}$ and oscillation friction was carried out at frequencies of 50 $\mathrm{Hz}$. The samples were washed with benzene and acetone in an ultrasonic bath before and after the friction test to remove contaminations and oil species for surface analysis. Table 2 summarizes the test condition of this paper.

2.4 The surface analysis with X-ray photoelectron spectroscopy (XPS) analysis

PHI Quantera II apparatus was used for XPS measurements to analyze the corresponding surfaces. The spot size for this analysis was $100 \mu \mathrm{m} \times 100 \mu \mathrm{m}$. Peak fitting and resolution were conducted with Shirley algorithm to estimate background and Gaussian-Lorentzian mixed function to separate each peak [5]. Chemical analysis as a function of depth with $\mathrm{Ar}$ ion etching was obtained on DLC and steel surfaces. When Ar ion etching was carried out, peak is considered to be shifted by the effect of charging. Hence, each spectrum is calibrated with Ar peak (241.7 $\mathrm{eV}$ ). Figure 2 is showing an example of XPS analysis of DLC surface. Figure 3 shows an example of XPS analysis for Molybdenum compound on steel surfaces.

The ratio of $\mathrm{sp}^{2} / \mathrm{sp}^{3}$ and $\mathrm{sp}^{2} /\left(\mathrm{sp}^{3}+\mathrm{C}-\mathrm{H}\right)$ was evaluated on DLC surfaces under the condition shown in Table 3. Ar ion etching was carried out in which the etching rates were approximately $8.8 \mathrm{~nm} / \mathrm{min}$ for DLC (calibrated with $\mathrm{SiO}_{2}$ ). In this report, the authors suppose that the spectrum of DLC is consisted of the peak of $\mathrm{sp}^{3}$ and $\mathrm{sp}^{2}$. The shift of $\mathrm{sp}^{2}$ carbon is detected at

Table 2 The detail of test condition

\begin{tabular}{c|c}
\hline \hline Friction tester & SRV (cylinder-on-disc) \\
Disc/substrate & DLC/SUJ2 (SAE52100) \\
Steel cylinder & $15 \mathrm{~mm}$ (dia) SUJ2 \\
Axial load & $100 \mathrm{~N}$ \\
Hertzian mean pressure (for steel pair) & $147.8 \mathrm{MPa}$ \\
Frequency & $50 \mathrm{~Hz}$ \\
Stroke length & $1.5 \mathrm{~mm}$ \\
Mean sliding speed & $0.15 \mathrm{~m} / \mathrm{s}$ \\
Temperature & $50^{\circ} \mathrm{C}$ \\
Test duration & $40 \mathrm{~min}$ \\
\hline
\end{tabular}

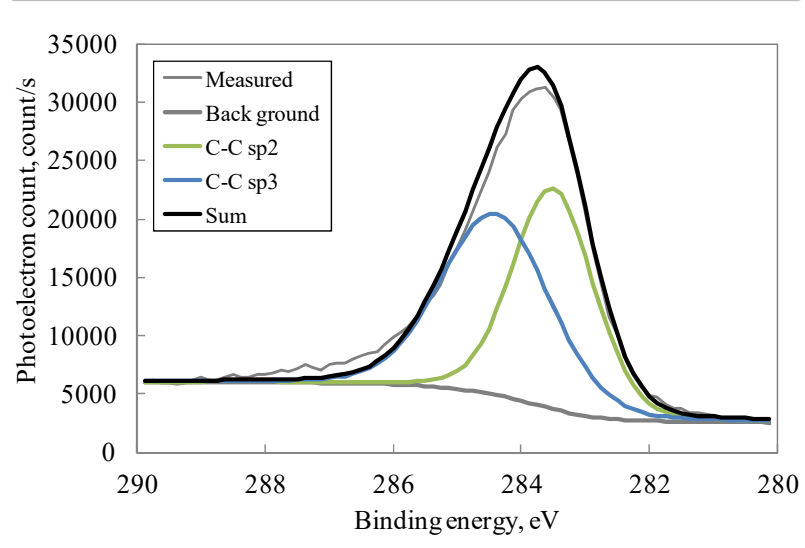

Fig. 2 Example of XPS for DLC coatings

Tribology Online, Vol. 12, No. 3 (2017) / 111 


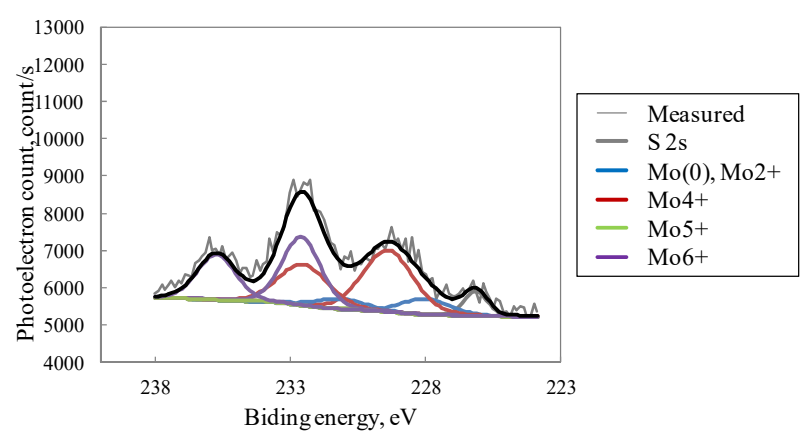

Table 3 The condition of peak resolution of XPS for DLC

\begin{tabular}{c|cc}
\hline \hline Peak & $\mathrm{sp}^{2}$ & $\mathrm{sp}^{3}$ \\
\hline Position [eV] & 284.3 & 285.2 \\
Position difference(from peak sp ${ }^{2}$ ) [eV] & - & 0.9 \\
Gaussian ratio [\%] & 80 & 80 \\
\hline
\end{tabular}

Fig. 3 Example of XPS for Steel

Table 4 The condition of peak resolution of XPS for Molybdenum compound

\begin{tabular}{|c|c|c|c|c|c|}
\hline Oxidation Number & 1 & 2 & 3 & 4 & 5 \\
\hline Name & $\operatorname{Mo}(0), 2+$ & Mo4+ & Mo5+ & Mo (0), 2+ & Mo4+ \\
\hline Orbit & $3 d 5 / 2$ & $3 d 5 / 2$ & $3 d 5 / 2$ & $3 d 5 / 2$ & $3 d 5 / 2$ \\
\hline Compounds & $\mathrm{Mo}, \mathrm{Mo}_{2} \mathrm{C}$ & $\mathrm{MoS}_{2}, \mathrm{MoO}_{2}$ & MoDTC & $\mathrm{Mo}, \mathrm{Mo}_{2} \mathrm{C}$ & $\mathrm{MoS}_{2}, \mathrm{MoO}_{2}$ \\
\hline Position $[\mathrm{eV}]$ & 228 & 229.4 & 231 & 231.13 & 232.53 \\
\hline Position difference (from peak1) $[\mathrm{eV}]$ & - & 1.4 & 3 & 3.13 & 4.53 \\
\hline Gaussian ratio [\%] & 80 & 80 & 80 & 80 & 80 \\
\hline Area ratio (from) & & & & $0.67(1)$ & $0.67(1)$ \\
\hline FWHM ratio (from) & & & & $1(1)$ & $1(2)$ \\
\hline Oxidation Number & 6 & 7 & 8 & 9 & \\
\hline Name & Mo6+ & Mo5+ & Mo6+ & $\mathrm{S}$ & \\
\hline Orbit & $3 d 5 / 2$ & $3 d 5 / 2$ & $3 d 5 / 2$ & $2 \mathrm{~s}$ & \\
\hline Compounds & $\mathrm{MoO}_{3}$ & MoDTC & $\mathrm{MoO}_{3}$ & & \\
\hline Position $[\mathrm{eV}]$ & 232.6 & 234.13 & 235.73 & $228 \pm 0.3$ & \\
\hline Position difference(from peak1) $[\mathrm{eV}]$ & 4.6 & 6.13 & 7.73 & & \\
\hline Gaussian ratio [\%] & 80 & 80 & 80 & 80 & \\
\hline Area ratio (from) & & $0.67(3)$ & $0.67(6)$ & & \\
\hline FWHM ratio (from) & & $1(3)$ & $1(6)$ & & \\
\hline
\end{tabular}

$284.3 \mathrm{eV}$ and the shift of $\mathrm{sp}^{3}$ ratio is detected at $285.2 \mathrm{eV}$ [6]. However, the shift of $\mathrm{sp}^{2}$ and $\mathrm{C}-\mathrm{H}$ are too close to separate each other. Hence the $\mathrm{sp}^{2} /\left(\mathrm{sp}^{3}+\mathrm{C}-\mathrm{H}\right)$ ratio is obtained on hydrogenated DLC [7].

The residue of molybdenum compound on the steel surfaces was evaluated with XPS under the condition shown in Table 4 [8]. Table 4 also shows the molybdenum compound which is presumed to exit [9]. Ar ion etching was carried out in which the etching rates were approximately $12.9 \mathrm{~nm} / \mathrm{min}$ for steel surfaces (calibrated with $\mathrm{SiO}_{2}$ ).

\section{Results}

3.1 The results of friction coefficient and specific wear rate Figure 4 shows friction coefficient of SRV sliding with each tribo pair. The friction coefficient of ta- $\mathrm{C}$ was lower than that of a-C:H under base oil lubrication. The friction coefficient of ta-C and a-C:H became lower with base oil + MoDTC and ta:C performed lower friction than a-C:H with base oil + MoDTC.

Figure 5 shows specific wear rate of DLC coated discs after 


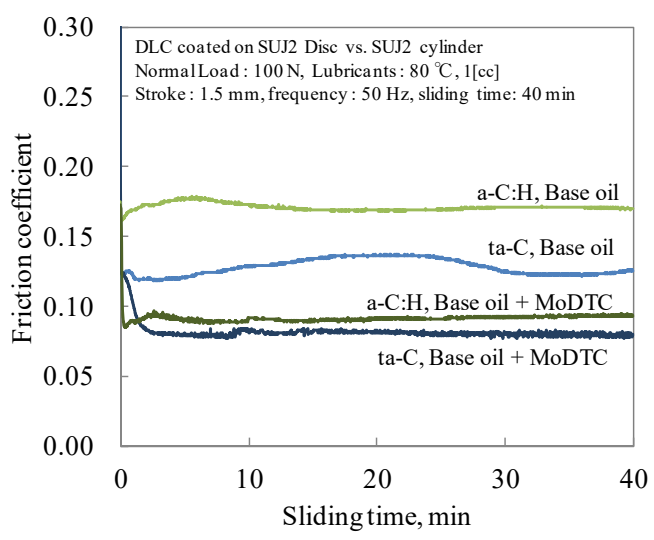

Fig. 4 The friction coefficient of SRV sliding with each tribo pair

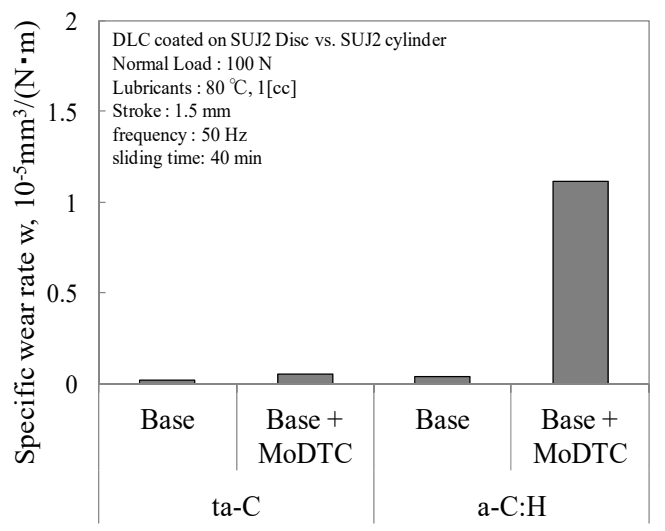

Fig. 5 Specific wear rate of DLC films

SRV sliding with base oil and base oil with MoDTC. Specific wear rate was calculated from cross sectional surface profile of wear track. Data obtained in Fig. 5 indicates that wear of ta-C after sliding with base oil was lower than wear of ta- $\mathrm{C}$ after sliding with base oil with MoDTC. On the other hand, a-C:H suffered huge wear after sliding with base oil with MoDTC comparing. As discussed previously [3], DLC containing hydrogen was easily worn under friction in the presence of MoDTC based lubricants.

\subsection{The analysis of DLC surface after sliding}

Figure 6 shows the optical microscope images after friction tests for ta-C and a-C:H coated discs and SUJ2 steel rollers as counterpart. In the case of ta-C coated discs in base oil with and without MoDTC as shown in Figs. 6(a,b), MoDTC did not show any difference of morphology for both of ta-C coated disc and SUJ2 steel. The wear scar of ta-C changed to smooth surface after friction test. On the other hand, in the case of a-C:H coated discs, MoDTC showed dark transferred particles on the rough wear scar. The wear scar of a-C:H without MoDTC in oil showed shinny smooth surface.

Figure 7 shows results of surface roughness $R_{a}$ of inside and outside of wear scars of DLC after friction test. Surface roughness Ra was obtained by surface profile-meter for DLC on the discs. On the surface of ta-C, Ra decreased after sliding and this tendency was similar between sliding with base oil and base oil with MoDTC. On the other hand, $R_{a}$ of a-C:H increased dramatically after sliding with base oil with MoDTC.

Structural analysis by using XPS of DLC was conducted and is shown in Figs. (8-11). DLC films are thought to be

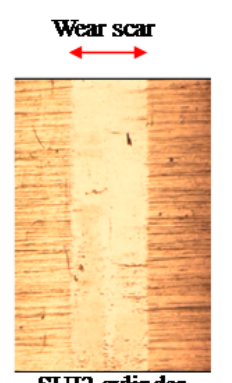

SUJ2 cylinder

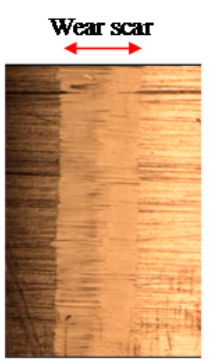

SUJ2 cylinder

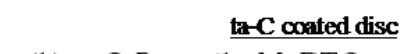

(b) ta C, Base oil + MoDTC

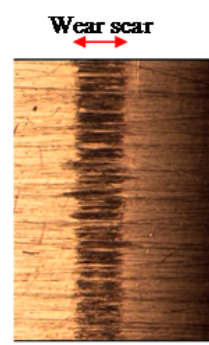

SUJ2 cylinder

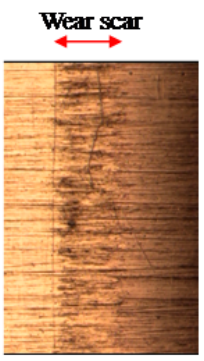

SUJ2 cylinder

(a) ta-C, Base oil

(c) a-C:H, Base oil

aC-H coated disc
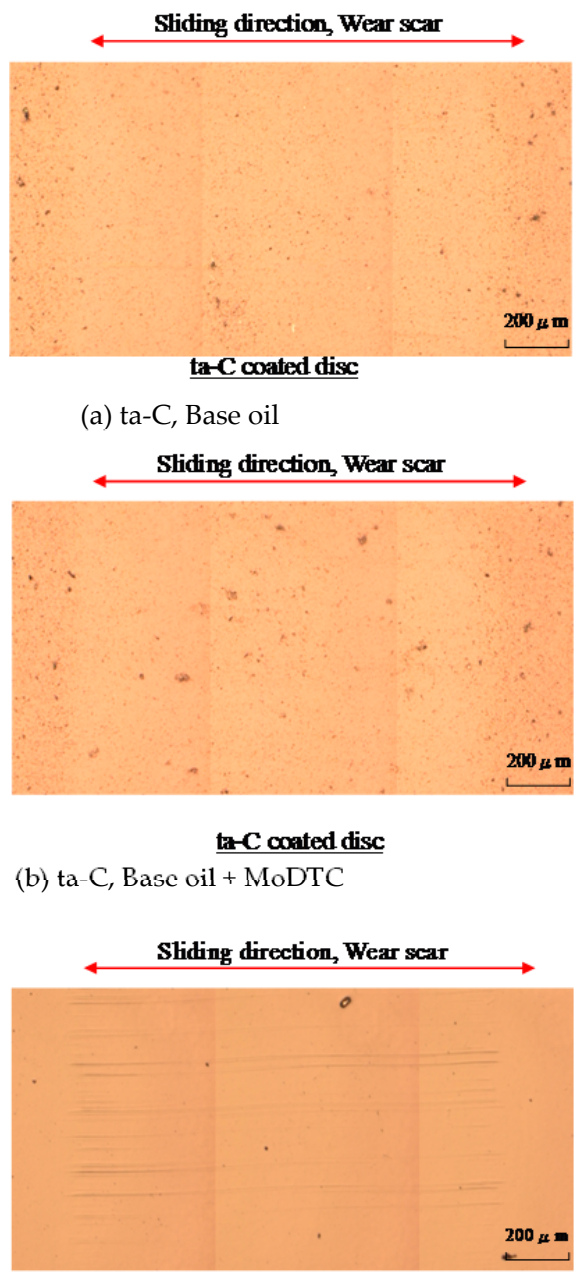

a-C:H coated disc

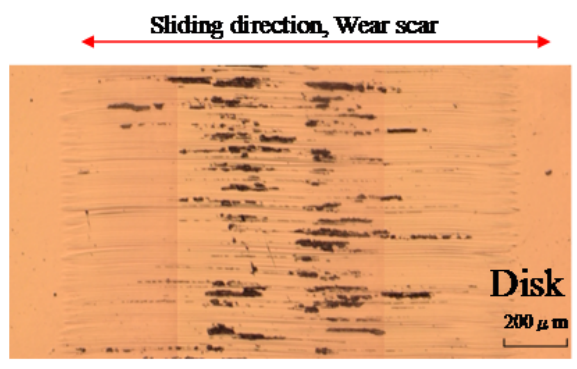

(d) a-C:H, Base oil + MoDTC

Fig. 6 Optical microscope images of wear scar of ta-C and a-C:H coated discs and SUJ2 cylinders with and without MoDTC in base oil and SUJ2 cylinders with and without MoDTC in base oil

predominantly amorphous in which small clusters of microcrystalline structure with $\mathrm{sp}^{3}$ and $\mathrm{sp}^{2}$ bonding, and amorphous matrix coexist $[10,11]$. It is thought that DLC could change its own structure from $\mathrm{sp}^{3}$ to $\mathrm{sp}^{2}$ with mechano-chemical reaction under friction. Sanchez-Lopez et al. reported that friction-induced structural transformations of diamond-like carbon affect friction coefficient under various atmospheres, in ambient air (RH s 30-40\%), dry air (RH-1\%), and dry nitrogen 


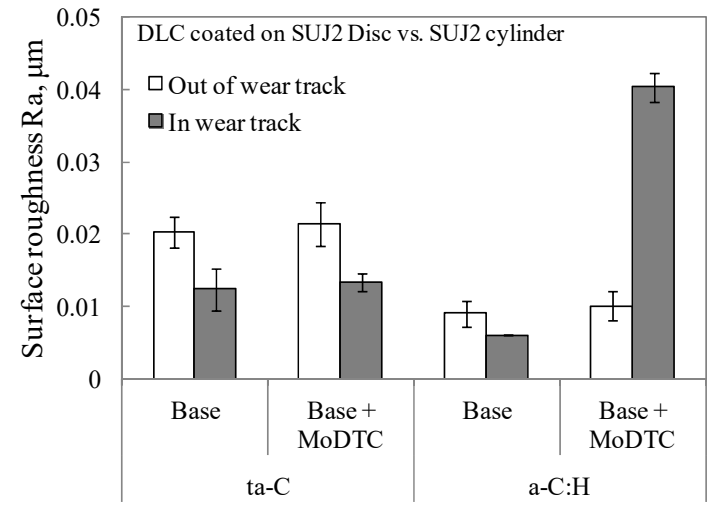

Fig. 7 Surface roughness of DLC films

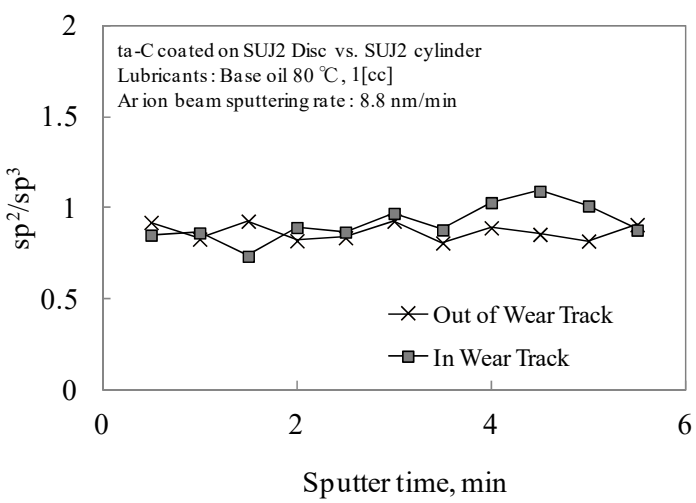

Fig. $8 \quad \mathrm{sp}^{2} / \mathrm{sp}^{3}$ ratio of ta-C film in sliding with base oil

$(0-1 \%)$ [12]. Mabuchi et al. found that DLC transformed graphite layer after sliding which is a consequence from friction under oil lubrication. They used transmission electron microscopy (TEM) and the thickness of transformed layer is a few nanometers thick [13]. There is a clear possibility that such transformed layer of DLC could affect tribological property of DLC. Therefore, the authors considered $\mathrm{sp}^{2}$ structural bonding states could play important role in DLC wear phenomena with MoDTC. Hence it is clearly important to evaluate the effect of MoDTC on the $\mathrm{sp}^{2} / \mathrm{sp}^{3}$ ratio of DLC surface. As can be seen in Figs. (8-11), the results of $\mathrm{sp}^{2}$ amount in DLC were calculated by the method of peak resolution on C1s with XPS. The authors conducted XPS analysis with $\mathrm{Ar}$ ion sputtering. The analysis enables the authors to consider the depth profile of structural transformation in the DLC films. The authors calculated $\mathrm{sp}^{2} /\left(\mathrm{sp}^{3}+\mathrm{C}-\mathrm{H}\right)$ as a vertical axis. ta-C does not have any hydrogen in its surface. Therefore $\mathrm{sp}^{2} / \mathrm{sp}^{3}$ was described as a vertical axis in Figs. (8,9). Only Fig. 10 shows $\mathrm{sp}^{2} /\left(\mathrm{sp}^{3}+\mathrm{C}-\mathrm{H}\right)$ among $0.5 \sim 2$ min sputtering time is higher than surface before sliding. However, it was not found that such $\mathrm{sp}^{2} /\left(\mathrm{sp}^{3}+\mathrm{C}-\mathrm{H}\right)$ increased in Fig. 11.

3.3 The result of tribochemical reacted compound on SUJ2 surface

Table 5 shows the analysis of chemical elements on SUJ2 roller surface. Figures $(12,13)$ show atomic concentration of Molybdenum with peak resolution analysis of XPS. Both figures describe that $\mathrm{Mo}^{6+}$ peak was rich on the surfaces, which was considered to come from $\mathrm{MoO}_{3}$. In addition, $\mathrm{Mo}^{2+}$, which is considered as $\mathrm{Mo}_{2} \mathrm{C}$ or Mo were found on the interface between

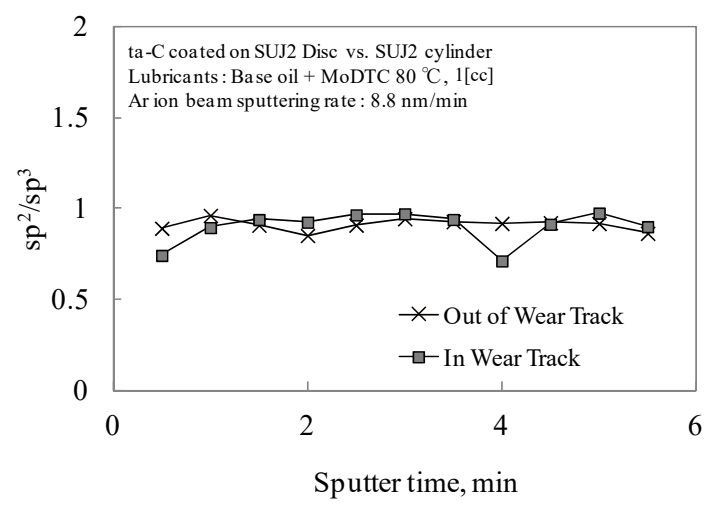

Fig. $9 \mathrm{sp}^{2} / \mathrm{sp}^{3}$ ratio of ta-C film in sliding with base oil + MoDTC

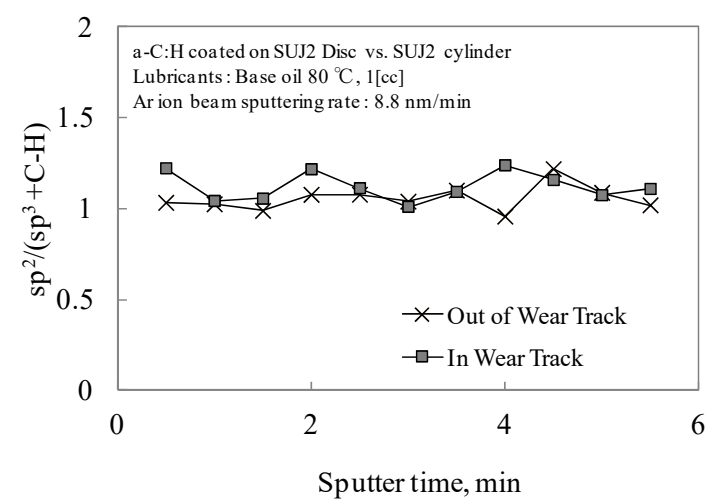

Fig. $10 \mathrm{sp}^{2} /\left(\mathrm{sp}^{3}+\mathrm{C}-\mathrm{H}\right)$ ratio of a-C:H film in sliding with base oil

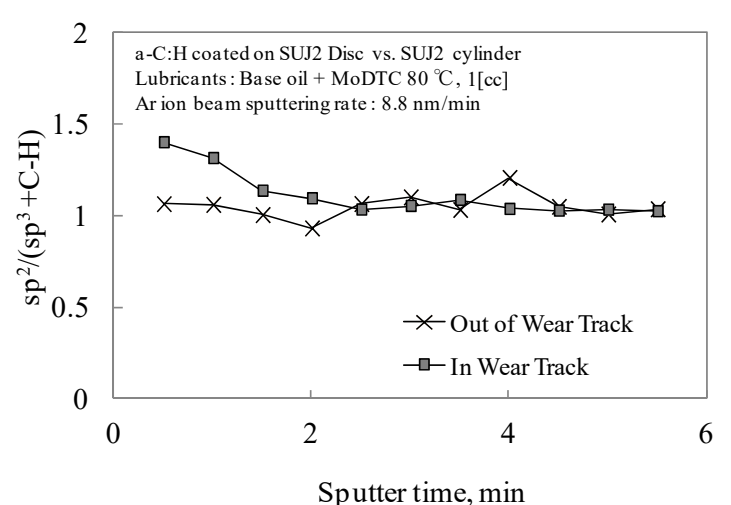

Fig. $11 \mathrm{sp}^{2} /\left(\mathrm{sp}^{3}+\mathrm{C}-\mathrm{H}\right)$ ratio of a-C:H film in sliding with base oil + MoDTC

tribofilm and substrate, SUJ2.

\section{Discussion}

Figure 5 shows that DLC, especially a-C:H suffered larger wear under lubrication with MoDTC. As prior studies has documented, the authors considered that the wear mechanisms of ta-C and a-C:H would be clearly different. Hence, in this study, the authors tested friction test with some simplified lubricants and conducted a detail analysis of tribofilm composition and DLC surfaces by XPS depth analysis.

XPS depth analysis - in Fig. 11 showing the structure of DLC surfaces - reveals that $\mathrm{sp}^{2} /\left(\mathrm{sp}^{3}+\mathrm{C}-\mathrm{H}\right)$ ratio becomes larger on 
Table 5 Atomic concentration on SUJ2 surface

\begin{tabular}{lc|ccccc}
\hline \hline \multirow{2}{*}{$\begin{array}{c}\text { Counter } \\
\text { DLC }\end{array}$} & Lubricant & \multicolumn{5}{|c}{ Atomic Ratio [\%] } \\
\cline { 3 - 7 } & Base oil & 6.1 & 4.8 & 0 & 89.1 & 0 \\
vs. ta-C & Base oil + MoDTC & 4.7 & 39.7 & 1.3 & 53.8 & 0.4 \\
& Base oil & 6.2 & 4.5 & 0 & 89.3 & 0 \\
\multirow{2}{*}{ vs. a-C:H } & Base oil + MoDTC & 7.6 & 38.5 & 2.0 & 50.7 & 1.3 \\
\hline
\end{tabular}

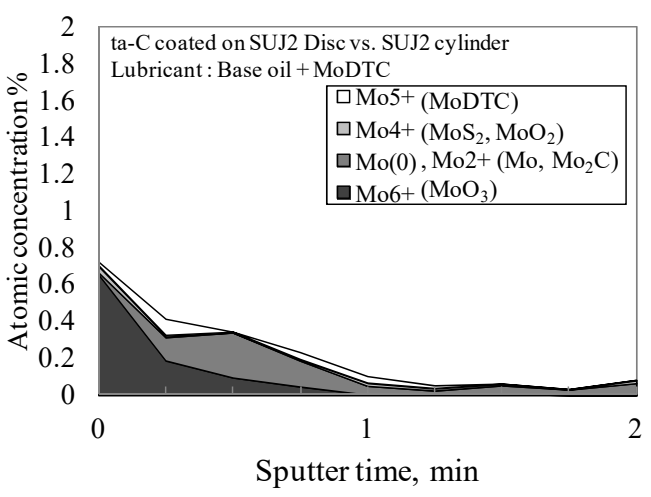

Fig. 12 Mo compounds depth profile on SUJ2 sliding with ta-C film

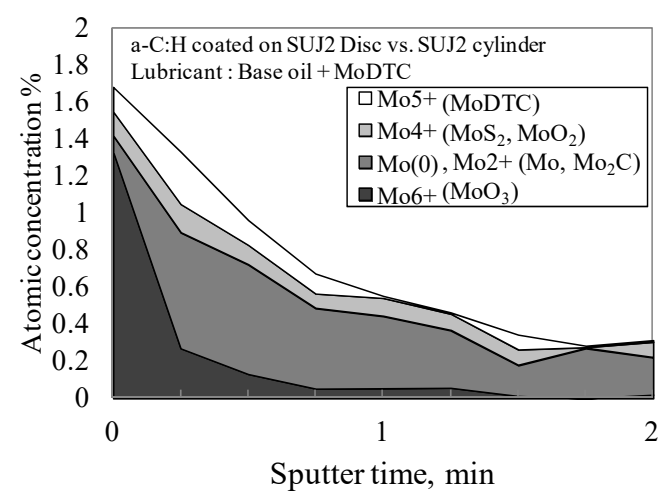

Fig. 13 Mo compounds depth profile on SUJ2 sliding with a-C:H film

a-C:H surface after sliding with lubricants with MoDTC. Huge wear also occurs on a-C:H surface in presence of MoDTC containing lubricants. This result indicates that MoDTC intervention on friction surfaces clearly accelerates increasement wear of a-C:H in association with structural transformation to $\mathrm{sp}^{2}$ from $\mathrm{sp}^{3}$ on the a-C:H surface.

In addition, surface roughness of ta-C after sliding with MoDTC was less than $R_{a}$ before sliding. However $R_{a}$ of a-C:H increased than $R_{a}$ before sliding as shown in Fig. 7. In general, such roughness smoothening would occur under chemical corrosion wear. Hence, the authors proposed chemical corrosion wear between ta-C surface and MoDTC occurred as a major factor in wear of ta-C. Because MoDTC generates $\mathrm{MoS}_{2}$ and $\mathrm{MoO}_{3}$ under friction surfaces by tribochemical reaction. The authors considered that carbon on ta-C surface would be reacted by oxidation with $\mathrm{MoO}_{3}$ and was removed as $\mathrm{CO}$ or $\mathrm{CO}_{2}$ as mentioned by Shinyoshi [3]. On the other hand, $R_{a}$ increment on
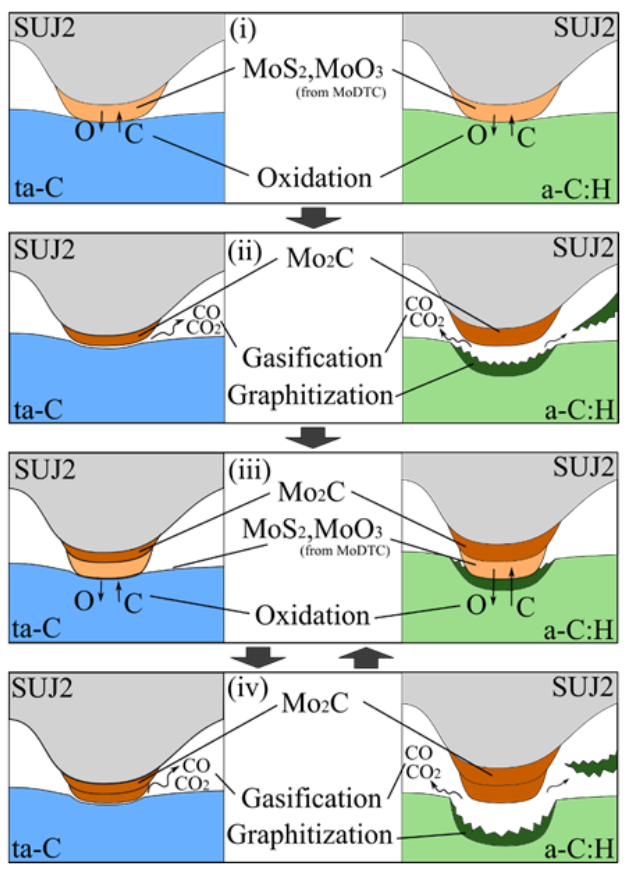

Fig. 14 Wear mechanism of DLC films by MoDTC

a-C:H and Molybdenum containing lubrication would seem to indicate mechanical wear after embrittlement by tribo-reaction with MoDTC and carbon on a-C:H surface.

The surface analysis with XPS of metal surface suggests that rich $\mathrm{MoO}_{3}$ was found in surface of tribofilm. In addition, rich $\mathrm{Mo}_{2} \mathrm{C}$ and $\mathrm{Mo}$ were found in interface between tribofilm and metal substrate. M. De Feo et al. reported that molybdenum compound was not observed on a-C:H surface but MoC was observed on steel surface of counter part of DLC by XPS. They considered that $\mathrm{MoC}$ was not so hard that DLC was worn by it. Therefore they considered that intermediate product of decomposed MoDTC reacts with DLC surface and then MoC was formed [14]. In this paper, the authors could observe the depth profile of tribofilm. This observation enables the authors to consider the order of chemical reaction between DLC and tribofilm of Molybdenum. The authors considered that $\mathrm{MoO}_{3}$ formed on the rubbed surface reacted by oxidation with carbon of DLC surface. After this oxidation, $\mathrm{MoO}_{3}$ changed to $\mathrm{Mo}_{2} \mathrm{C}$ or $\mathrm{Mo}$ and remained in tribofilm on SUJ2 surface by the extreme high stress and heat under friction.

The authors proposed the wear model to explain the mechanism about DLC wear with MoDTC based lubricant as shown in Fig. 14. In the authors' hypothesis, the mechanism 
consists of four elements below.

(i) MoDTC was decomposed on friction surfaces and generated $\mathrm{MoS}_{2}$ and $\mathrm{MoO}_{3}$ tribofilm on the surface asperities. Oxygen from $\mathrm{MoO}_{3}$ layer in this tribofilm and carbon from DLC surface would be mixed each other and form interlayer between tribofilm and DLC surface.

(ii) Gasification to $\mathrm{CO}$ and $\mathrm{CO}_{2}$ from oxidation of $\mathrm{MoO}_{3}$ and DLC would occur because the stress under friction causes to break $\mathrm{C}-\mathrm{C}$ bonding. Activated carbon atoms during friction would react with oxygen of $\mathrm{MoO}_{3}$. In ta-C wear condition, this gasification would be a major factor. The hardness of ta-C is originally higher and should be enough to resist mechanical wear and only surface asperities are removed by this oxidation. It could contribute to smooth ta- $C$ surface after molybdenum based lubrication. On the other hand, in a-C:H, structural transformation to $\mathrm{sp}^{2}$ from $\mathrm{sp}^{3}$ occurs in addition to the oxidation. The hydrogen of a-C:H would play an important role in the mechanism of structural transformation to $\mathrm{sp}^{2}$ from $\mathrm{sp}^{3}$. C-H site is exposed to outside of carbon structure of DLC and more easily react $\mathrm{MoO}_{3}$ than C-C. Activated carbon atom recombined with other carbon atoms. Hence $\mathrm{sp}^{2}$ hybridization status is considered to increase. Microscopically, it could be assumed that $\mathrm{sp}^{2}$ rich site in DLC structure is easily destroyed and embrittlement of a-C:H would proceed. This structural change in locally on surface would result in microscopically local wear on a-C:H surface in associated with adhesion and shear force under frictional surfaces. It is clear that such local wear could result in surface roughness increment. In both of these ta- $\mathrm{C}$ and a-C:H lubrication systems, $\mathrm{MoO}_{3}$ could be changed into $\mathrm{Mo}_{2} \mathrm{C}$ by reduction action.

(iii) $\mathrm{Mo}_{2} \mathrm{~S}$ and $\mathrm{MoO}_{3}$ generate on $\mathrm{Mo}_{2} \mathrm{C}$ residue from $\mathrm{MoDTC}$ decomposition and carbon and oxygen expand between $\mathrm{MoO}_{3}$ layer and DLC surface.

(iv) As been described in (ii), wear of ta-C proceed by chemical corrosion with gasification as a major factor. Wear of a-C:H would be accelerated due to structural transformation to $\mathrm{sp}^{2}$ from $\mathrm{sp}^{3}$ on its surface. Then the situation would back to the status as (iii).

\section{Conclusion}

In order to clarify the mechanism of DLC wear by MoDTC based lubricants, the authors conducted the friction tests with ta-C and a-C:H under lubrication of base oil and based oil containing MoDTC. The surface analysis of DLC and metal surface was also conducted. The previous research has revealed that MoDTC increases wear of DLC under contact between steel and DLC and tribochemistry of Mo compound on the surfaces play an important role to explain this phenomenon. In this paper, the authors revealed that the distribution of Mo compound in the tribofilm on the metal surface with the depth profile analysis by XPS. This result contributes to understand oxidation-reduction reaction under contact between DLC and Steel in Mo based lubricants. The authors revealed that Mo in the outermost surface of tribofilm was reduced by carbon of DLC than that in deeper region of tribofilm. As a result, the authors consider that it is necessary that $\mathrm{Mo}_{2} \mathrm{C}$ or Mo generates in tribofilm when wear of DLC increase. The authors believe that these specific structure of tribofilm on the counterpart surface of steel ball against a-C:H disc is quite important to provide severe wear of a-C:H. The conclusions below were obtained experimentally.
(1) There were small wear on ta-C and huge wear on a-C:H under lubrication with base oil containing MoDTC.

(2) Structure of ta-C surface was unchanged by lubrication with base oil containing MoDTC. The roughness of the surface of ta- $\mathrm{C}$ decreased than surface before sliding. On the other hand, structure of a-C:H surface transformed to rich $\mathrm{sp}^{2}$ ratio structure and this surface has larger roughness compared with before sliding.

(3) The analysis on SUJ2 surface revealed that $\mathrm{MoO}_{3}$ was the majority on the outermost surface and $\mathrm{Mo}_{2} \mathrm{C}$ and Mo were observed on the vicinity of SUJ2 substrate.

On the basis of these three experimental results, the authors proposed the hypothesis about DLC wear mechanism with MoDTC based lubricants as described in Fig. 14. The authors have considered that $\mathrm{MoO}_{3}$ is formed from MoDTC decomposition under contact surfaces and would react with carbon of DLC surfaces substrate. If this reaction results in structural transformation to $\mathrm{sp}^{2}$ from $\mathrm{sp}^{3}$ as can be seen in a-C:H tribo-pairs, mechanical wear would accelerate wear of DLC.

\section{Refernces}

[1] Robertson, J., "Diamond-Like Amorphous Carbon," Materials Science and Engineering, 37, 4-6, 2002, 129-281.

[2] Vižintin, J., Kalin, M., Dohda, K. and Jahanmir, S., "Tribology of Mechanical Systems," ASME Press, New York, 2004, 139-156.

[3] Shinyoshi, T., Fuwa, Y. and Ozaki, Y., "Wear Analysis of DLC Coating in Oil Containing Mo-DTC," SAE Technical Paper, 2007-01-1969, 2007.

[4] De Barros, M. I., "Boundary Lubrication Mechanisms of Carbon Coatings by MoDTC and ZDDP Additives," Tribology International, $38,3,2005,257-264$.

[5] Ansell, R., Dickinson, O., Povey, T. A. F. and Sherwood, P. M. A., "X-Ray Photoelectron Spectroscopic Studies of Electrode Surfaces Using a New Controlled Transfer Technique," Journal of Electroanalytical Chemistry and Interfacial Electrochemistry, 98, 1, 1979, 79-89.

[6] Dias, J., Paolicelli, G., Ferrer, S. and Comin, F., "Separation of $\mathrm{sp}^{3}$ and $\mathrm{sp}^{2}$ Components in the $\mathrm{C} 1 \mathrm{~s}$ Photoemission Spectra of Amorphous Carbon Films," Physical review B, 54, 1996, 8064-8069.

[7] Tokoroyama, T., Hatano, T., Umehara, N. and Fuwa, Y., “The Effect of Ultraviolet Light Irradiation on Tribological Properties of Hydrogenated DLC," Micro-NanoMechatronics and Human Science (MHS), 2010, 234-238.

[8] Grim, S. O. and Matienzo, L. J., "X-Ray Photoelectron Spectroscopy of Inorganic and Organometallic Compounds of Molybdenum," Inorganic Chemistry, 14, 5, 1975, 1014-1018.

[9] Thompson, A. (Editor), "X-RAY DATA BOOKLET," University of California, Berkeley, Barkeley, 2009.

[10] Spencer, E. G., Schmidt, P. H., Joy, D. C. and Sansalone, F. J., "Ion-Beam-Deposited Polycrystalline Diamondlike Films," Applied Physics Letters, 29, 2, 1976, 118-120.

[11] Joy, D. C., Spencer, E. G., Schmidt, P. H. and Sansalone, F. J., "The Structure of Ion-Deposited Crystalline Carbon Films," Proceedings of 34th Annual Meeting of Electron Microscopy Society of America, 1976, 646.

[12] Sanchez-Lopez, J. C., Erdemir, A., Donnet, C. and Rojas, T. C., "Friction-Induced Structural Transformations of Diamondlike Carbon Coatings under Various Atmospheres," Surface and Coatings Technology 163-164, 2003, 444-450.

[13] Higuchi, T., Mabuchi, Y., "Structure Transformation of Hydrogen Free DLC Surface with Friction," Proceedings of JAST Tribology Conference Tokyo, 2011, 135.

[14] Feo, M. De., Bouchet, M. I. D. B., Minfray, F., Esnouf, C., Mogne, T. L., Meunier, F., Yang, L. Q., Thiebaut, B., Martin, J., M., “Wear Mechanism of H-DLC under Lubrication with MoDTC-Containing Base Oil," Proceedings of ITC 2015 Tokyo, 2015, 808-809. 